Cahiers d'études africaines

\title{
Paroles de papier. Matérialité et écritures en contextes africains
}

Rémi Dewière et Silvia Bruzzi

\section{(2) OpenEdition \\ 1 Journals}

Édition électronique

URL : https://journals.openedition.org/etudesafricaines/27281

DOI : 10.4000/etudesafricaines. 27281

ISSN : $1777-5353$

Éditeur

Éditions de l'EHESS

Édition imprimée

Date de publication : 5 décembre 2019

Pagination : 949-966

ISBN : 9782713227813

ISSN : 0008-0055

Référence électronique

Rémi Dewière et Silvia Bruzzi, «Paroles de papier. Matérialité et écritures en contextes africains », Cahiers d'études africaines [En ligne], 236 | 2019, mis en ligne le 05 décembre 2019, consulté le 07 janvier 2022. URL : http://journals.openedition.org/etudesafricaines/27281 ; DOI : https://doi.org/ 10.4000/etudesafricaines. 27281 


\section{Paroles de papier}

\section{Matérialité et écritures en contextes africains*}

Les supports de l'écrit sont devenus un objet d'étude à part entière. En euxmêmes, ainsi que dans leur articulation avec le ou les signes qui y sont inscrits, ils révèlent des discours, des pratiques et des interactions sociales complexes et aux ramifications temporelles et géographiques potentiellement très vastes. L'intérêt pour les supports écrits s'insère dans l'explosion des régimes de matérialité, en particulier dans le large recours aux dispositifs de numérisation des ressources textuelles manuscrites et imprimées à l'échelle mondiale et de la démocratisation de leur consultation. En effet, le mouvement de numérisation, initié depuis une vingtaine d'années ${ }^{1}$, a atteint une maturité qui permet d'engager une réflexion à la fois sur la matérialité de l'écrit, à travers ses supports, mais également sur le rapport sensible que l'on entretient avec ces documents. En effet, la matérialité scripturaire n'est pas seulement accessible par une approche codicologique des supports physiques et de leur propriété. Elle doit aussi être envisagée par les effets sensibles et ressentis des usages d'un support. Si, d'un côté, la numérisation des documents a relancé les questionnements sur les procédés matériels de production de l'écrit, de l'autre les vecteurs numériques et leur visualisation à l'écran peuvent également être considérés

*. Ce numéro est le résultat d'une collaboration scientifique entre l'Institut des Mondes Africains à Paris et le Centre for Middle Eastern Studies de l'Université de Bergen. Initiée en 2014, dans le cadre du Partenariat Hubert Curien (PHC) franco-norvégien, la collaboration a réuni des chercheurs de différentes spécialités et disciplines autour de la matérialité des textes dans l'histoire de l'Afrique, du Moyen Âge à l'époque contemporaine, et sur les usages, les modalités de fabrication et de circulation des documents écrits, quels que soient leurs supports. Le point d'orgue de ce numéro fut une table ronde internationale organisée, le 15 mars 2017 à l'IMAf (Paris), en collaboration avec le SMI de l'Université de Bergen (Norvège). Nous souhaitons remercier Anne Bang, Anouk Cohen, Ghislaine Lydon, Didier Nativel et Alexandros Tsakos, ainsi que les relecteurs anonymes, pour leurs commentaires.

1. Le site internet Gallica fut créé en 1997 ; puis suivirent les sites du programme Endangered Archives (2004), Google Livres (2004) ou Archive.org (1996 pour les pages internet; 2005 pour les livres imprimés), pour citer quelques initiatives privées et publiques.

Cahiers d'Études africaines, LIX (4), 236, pp. 949-966. 
comme une couche supplémentaire dans le rapport sensible entre le lecteur et un texte. Ainsi, les formes numériques de communication réorganisent les informations, tout en ajoutant de nouvelles à travers l'association et/ou la dissociation des formes et des matériaux (Bonaccorsi 2013 : 127). Dès lors, il nous a semblé pertinent d'engager une réflexion historique et anthropologique qui ne parte pas du contenu narratif des textes, mais de leurs supports, afin de comprendre les sociétés de production et de réception de ces textes, mais également notre propre rapport, en tant que chercheurs, à l'objet écrit.

Cette réflexion est le point de départ de ce numéro consacré à la matérialité de l'écrit en contextes africains. Elle s'inscrit dans le renouveau des études historiques et anthropologiques sur l'écrit en Europe et, ces vingt dernières années, en Afrique. Dans la foulée du tournant archivistique qui a vu « le passage de l'archive-comme-source à l'archive-comme-sujet » (Stoler 2019 : 78, voir aussi Jungen \& Raymond 2012), l'objet écrit n'a plus été cantonné à son statut de source, mais il est devenu un sujet propre. Le «tournant matériel» (material turn) fut décisif pour requestionner les supports papiers de l'écrit en tant qu'objets (Roche 1997 ; Hall 2000 ; Stolow 2010 ; Van Damme 2015), qui étaient «en grande partie demeurés pour les chercheurs sans grand intérêt» (Hébrard 1999: 10, cité dans Mbodj-Pouye 2009: 857). Dans les traces de Guglielmo Cavallo et d'Armando Petrucci, Roger Chartier et Christian Jacob ont eu un rôle séminal dans les études de l'écriture et de la lecture en Europe ${ }^{2}$, et de nombreux chercheurs en études africaines se sont directement inspirés de ses travaux, des anthropologues (Cohen 2016 ; Mbodj-Pouye 2013) aux historiens (Peterson 2004). En parallèle, la relation entre sens, sensualité et objets écrits tels les lettres, a fait l'objet d'une attention particulière ces dernières années, en particulier dans le champ de l'histoire globale et de la micro-histoire (Raabke 2017 ; Freist à paraître : 75). Les réflexions de ce champ relativement neuf commencent également à alimenter le champ des études africaines, comme le montrent plusieurs contributions à ce numéro.

À l'image de l'Europe (Anheim \& Chastang 2009), l'étude de la culture de l'écrit en Afrique s'appuie sur une tradition érudite plus ancienne, où la paléographie a tenu une place importante (Ficquet \& Mbodj-Pouye 2009 : 753;

2. La bibliographie sur le sujet est très vaste. On peut toutefois signaler les travaux précurseurs de F. Furet \& J. Ozouf (1977), de G. Cavallo (1983), d'A. Petrucci $(1979,2008)$ et de R. Chartier (1985, 1987a, b, 1991). Le dossier spécial des Annales intitulé «Pratiques d'écriture. Une histoire de la culture écrite» (vol. 56, n 4-5, 2001) rend compte des avancées réalisées sur l'histoire de l'Europe dans le dernier quart du $\mathrm{XX}^{\mathrm{e}}$ siècle. D'autres ouvrages, comme Lieux de savoir 2. Les mains de l'intellect, dirigé par C. ЈАСОВ (2011), et The Nature of the Book, d'A. JoHns (1998), sont devenus des références incontournables. 
Wion et al. 2016) ${ }^{3}$. Or, depuis les travaux pionniers de Rex Sean O'Fahey et John Hunwick sur la littérature arabe en Afrique (O’Fahey \& Abu Salim 1983 ; Hunwick 2005), une branche importante d'études s'est développée sur les cultures manuscrites africaines (Krätli \& Lydon 2011 ; Delmas \& Penn 2012 ; Bausi et al. 2015 ; Brigaglia \& Nobili 2017). Grâce à la collecte et à la valorisation d'une riche littérature manuscrite endogène, plusieurs projets ont développé une perspective d'analyse attentive aux interactions entre écriture, visualité et oralité, aux échanges entre différentes cultures manuscrites (chrétiennes et islamiques, notamment) jusqu'aux pratiques de collecte et d'archivage.

Par ailleurs, le continent africain a rarement été pris en compte par les historiens du livre. Ces derniers ont souvent souligné le rôle des missionnaires, des administrateurs coloniaux (Davis, Dick \& Le Roux 2018 : 377) et des commerçants européens ou arabes dans la diffusion du livre et de la literacy en Afrique subsaharienne (Krätli \& Lydon 2011). C'est récemment que l'histoire du livre, de la littérature et de la presse en Afrique subsaharienne a retenu l'attention des chercheurs (Le Roux 2012), qui se sont intéressées aux premiers écrits endogènes en Afrique (Ricard 1995 ; Van Dalen 2016 ; Delmas 2017 ; Dewière 2017) aux processus contemporains de formation d'un public de lecteurs, à l'impact social d'une culture littéraire, à la production et circulation des livres dans des circuits transnationaux (Mirza 2017), notamment en Égypte et en Afrique du Sud (Yusuf 2016; Davis, Dick \& Le Roux 2018). L'histoire de la culture de l'imprimé en Afrique a soulevé les questions de genre et de classe, notamment en Égypte où les femmes sont rentrées, dès la fin du XIX ${ }^{\mathrm{e}}$ siècle, dans l'espace masculin de la presse, en acquérant une nouvelle visibilité sociale et en tant qu'écrivaines et lectrices des journaux, poèmes et autobiographies (Booth 2001 ; Yusuf 2016).

En France, les recherches historiques et anthropologiques en Afrique se sont emparées de la question de l'écrit, de ses usages à sa production, en passant par son archivage, ouvrant la voie à l'étude de l'objet écrit, ainsi qu'à la manière dont il a été fabriqué et aux outils utilisés, pour en tirer des informations sur le passé et le présent des sociétés africaines. Le dossier des Annales, coordonné par Éloi Ficquet et Aïssatou Mbodje-Pouye (2009), consacré aux cultures écrites en Afrique, fut une étape importante de la recherche sur les sociétés africaines en France. En se concentrant principalement sur la production écrite issue de la rencontre coloniale et des périodes coloniales et post-coloniales, le dossier a posé les jalons d'une recherche qui a influencé dans ses questionnements

3. Nous tenons à souligner l'importance de la codicologie et de la paléographie des textes en langue copte et arabe, pour laquelle il existe une vaste littérature centrée sur l'Afrique du Nord et le Moyen-Orient (DÉROCHE 2000). 
des spécialistes de toutes les aires géographiques, et de toutes les périodes historiques. En 2016, le dossier spécial coordonné par Anaïs Wion, Sébastien Barret et Aïssatou Mbodj-Pouye, a apporté une pierre supplémentaire au débat, en se concentrant sur un type de document peu pris en considération par les spécialistes de l'écrit en Afrique : les écrits pragmatiques. L'attention portée à ces écritures non littéraires, à savoir « un registre d'écriture lié à l'exercice des fonctions administratives et juridiques » (Wion, Barret \& Mbodj-Pouye 2016), a mis en avant la relation étroite entre pouvoir et écrit, ainsi que les enjeux autour de la conservation et de l'archivage des textes du pouvoir (Street 1993 ; Goody 2007 ; Mbodj-Pouye \& Fraenkel 2010).

Le présent numéro s'inscrit dans la continuité de ces travaux. L'originalité des contributions tient dans leur focale, puisqu'elles ne partent pas du contenu narratif des documents écrits, mais de tous les éléments qui entourent l'artefact scripturaire en tant qu'objet : le support, mais aussi les gloses, l'aspect graphique ou la relation entre le texte et son environnement à travers les sens. En découvrant un manuscrit, une inscription épigraphique, un acte juridique ou une photographie, le chercheur fait face à un objet qui a été spécialement pensé, organisé et produit pour s'inscrire dans une interaction concrète entre les originaires et les destinataires (Stollberg-Rilinger 2008). Leur analyse révèle les faits sociaux qui peuvent être analysés dans les traits matériels des documents écrits et qui seraient restés silencieux si l'on ne s'attachait qu'aux traits discursifs. Se concentrer sur la matérialité des documents écrits ouvre de nombreuses perspectives de recherche. Cela permet d'analyser les messages exprimés à travers les supports et leurs interactions avec leur contenu textuel en décryptant la mise en page, les images et les styles d'écriture choisis par leurs auteurs et les copistes. Croisées avec l'étude textuelle des documents, les articulations entre formes et matériaux peuvent mettre en lumière des messages complémentaires, des sous-textes et des histoires inédits, restituant ainsi une histoire plus complexe des sociétés africaines présentes ou passées.

La question de l'interaction entre matérialité et discours pose également un défi méthodologique. À l'aide de réflexions déjà menées en Afrique ou dans d'autres aires géographiques (Chartier 1991 ; Fabre 1993 ; Goody 2007 ; Denis \& Pontille 2012; Mbodj-Pouye 2013; Thyrza Sparks 2013), nous souhaitons interroger le rapport des historiens et des anthropologues de l'Afrique avec leurs sources, à travers une réflexion méthodologique nouvelle. Ce questionnement peut être le résultat d'une approche pragmatique, liée à la nature parcellaire de la documentation écrite, mais aussi d'un besoin de multiplier les niveaux d'analyse (de l'histoire sociale, politique et économique à l'histoire des techniques, des usages quotidiens ou bureaucratiques). Ainsi, les recherches sur la matérialité de l'écrit permettent de pallier les lacunes de nos sources, et de mettre en évidence 
ses acteurs de l'ombre, comme les scribes, les imprimeurs ou les simples lecteurs. Enfin, la prise en compte du matériau dans l'analyse historique construit un lien fort entre l'historien et les personnes qui conçurent et/ou manipulèrent le document étudié. Des mains de l'artisan à celles du chercheur, la lettre, la stèle ou la photographie ont transmis autant d'émotions qu'elles ont rencontré d'intermédiaires, et se sont chargées de leur mémoire et de leur expérience.

\section{Le papier et l'écrit, au sens le plus concret}

Les matériaux et les formats de l'écrit sont nombreux, du roc au sable (Lefebvre 2009), de la peau (Vaughan 2007) aux tissus (Parkin 2005) ou de l'argentique aux écrans. Toutefois, un support a retenu l'attention de l'ensemble des contributions : le papier. Si d'autres supports ont toujours coexisté, du papyrus aux murs, en passant par les feuilles ${ }^{4}$, le papier et ses contraintes économiques et logistiques sont un facteur essentiel à prendre en compte dans l'histoire des institutions économiques, religieuses, juridiques et politiques en Afrique (Lydon 2009 : 648-649), et ce jusqu'à aujourd'hui. En se concentrant sur le papier ou, pour les périodes les plus anciennes, sur sa cohabitation avec le papyrus et le parchemin, nous avons décidé de restreindre le champ d'analyse pour explorer la pluralité d'approches et d'enjeux autour de l'un des supports de l'écrit les plus répandus et partagés dans le temps et dans l'espace africain.

Le papier est plus que le simple support de la parole écrite. Il l'accompagne dans les « deux âges » de la vie du document, celui de sa conception et réalisation et celui de son voyage (Morelle 2009: 117). Toutefois, de la fabrication du papier dans les usines de Méditerranée et d'Europe du Nord à la conception du document écrit en Afrique, l'histoire du papier est une histoire des circulations et des échanges économiques de longue distance. À notre connaissance, il n'existe pas de production locale de papier et celui-ci est importé depuis le nord du Sahara jusqu'à la fin du XIX ${ }^{\mathrm{e}}$ siècle, ce qui en fait un produit rare et cher (Bloom 2008 : 45). Pourtant, le papier pénètre rapidement en Afrique subsaharienne. Né en Chine, le papier est introduit en Méditerranée à travers le califat abbasside, au VIII ${ }^{\mathrm{e}}$ siècle (ibid. : 9). Dès le $\mathrm{X}^{\mathrm{e}}$ siècle, $\mathrm{il}$ est utilisé dans le cadre du commerce transsaharien : le géographe arabe Ibn Hawkal mentionne un instrument de dette, identifié comme un chèque (șakk), de 42000 dinars à Awdaghost au $\mathrm{X}^{\mathrm{e}}$ siècle, signé par des témoins (ibid. : 138 ; Lydon dans ce numéro).

4. Un champ particulièrement important concerne l'épigraphie médiévale (DE MORAES Farias 2004 ; Fauvelle, Hirsch \& CheKroun 2017 ; Derat 2018). 
Comme le remarque Ghislaine Lydon dans son article, ce document fut probablement rédigé sur papier, mais il n'est pas impossible que du papyrus ou du parchemin aient été utilisés. À cette époque, la production de papier vient concurrencer le papyrus et le parchemin en Méditerranée et au sud du Sahara. La transition du parchemin et du papyrus au papier est également décrite par Alexandros Tsakos qui montre dans son article que leur coexistence implique des usages et des sensibilités différenciés : le cuir était surtout utilisé pour les documents légaux, le parchemin pour les textes littéraires et liturgiques et le papier pour la correspondance (Ochała 2014 : 14). Or, cette coexistence cesse rapidement et l'usage du papier se généralise, bien qu'il fasse l'objet de certaines résistances : au Maghreb médiéval pour l'écriture des textes religieux (Déroche 2005 : 35), dans les institutions coloniales du Togo (Glasman 2012) ou encore dans les échanges monétaires, comme dans le cas des Nuer (Hutchinson 1996). De l'Antiquité au XIVe siècle, les « instruments de papier », tels que les définit Ghislaine Lydon dans son article, sont produits en Afrique du Nord et alimentent les marchés sahéliens, nubiens et éthiopiens. La fin du Moyen Âge voit s'opérer un glissement majeur de la production de papier vers le monde chrétien. Les papiers espagnols, puis français et surtout italiens envahissent la Méditerranée avec le tre lune, un papier dont la contremarque est spécialement créée pour le marché islamique (Humbert 2001 : 211). La production de papier d'Afrique du Nord s'effondre et le papier italien est la principale source de papier au Sahel jusqu'au début du XX $x^{e}$ siècle (Walz 1985 ; Bloom 2008) : de nombreuses lettres diplomatiques étudiées par Rémi Dewière sont rédigées sur un tel papier, même pendant l'occupation britannique. L'aire de diffusion du tre lune est large, puisque plusieurs documents montrent qu'il est utilisé jusqu'au nord du Ghana, au côté de papier oriental (Levtzion 1965 : 118).

Jusqu'au XIX ${ }^{\mathrm{e}}$ siècle, les mentions du commerce de papier sont rares, bien que de nombreux témoignages, ainsi que la présence d'une littérature endogène dans la Corne de l'Afrique ou au Sahel attestent d'un usage régulier (Bloom 2008 : 53). Au XIX siècle, les premiers témoignages européens de l'intérieur de l'Afrique de l'Ouest permettent de faire des estimations sur la quantité du papier, sur son économie, mais aussi sur la circulation du papier européen (Walz 1985 ; Last 2008 ; Nobili 2011) et non européen (Regourd 2018). Trois observations viennent nuancer la rareté et la cherté du papier : il est présent sur de vastes portions du continent, quand bien même les quantités seraient dérisoires ; il s'agit d'un bien certes cher, mais qui n'est pas exorbitant et, enfin, les prix varient grandement en fonction du lieu d'achat. Ainsi, en 1767, Tripoli exportait deux mille rames de papier vers le Sud, ce qui équivalait à quatre millions de folios (Last 2008 : 152). Si le papier est une 
denrée commune dans les grandes villes, comme à Tombouctou, Harrar, Kano ou Kukawa, on peut trouver quelques feuilles sur les étals des marchés les plus reculés. Toutefois, les approvisionnements peinent à satisfaire la demande, et les lettrés vivant aux marges des zones de chalandise des grandes villes sahéliennes cherchent des voies d'approvisionnement alternatives (Levtzion 1965 : 119), ou d'autres supports. Cette difficulté à trouver du papier de qualité ou bon marché, en fonction des besoins, perdure dans certains endroits jusqu'à la seconde moitié du XXe siècle (Mutai \& Brigaglia 2017).

L'expansion coloniale en Afrique s'accompagne de deux technologies qui révolutionnent les usages de 1'écrit : la machine à papier en continu et l'imprimerie. Inventée en 1799, la machine à papier en continu est massivement industrialisée dans la première moitié du XIX ${ }^{\mathrm{e}}$ siècle. Le papier en rouleau remplace rapidement le papier à cuve italien et il inonde le marché africain à partir des côtes (Lamberg et al. 2012 : 11), le papier devenant l'un des principaux outils de pouvoir des administrateurs coloniaux ${ }^{5}$. En Afrique de l'Est, le papier britannique est exporté par des marchands indiens, ce qui souligne l'extraordinaire complexité de ses circuits (Regourd 2018). Si le papier est alors étroitement lié au pouvoir colonial, il est également recyclé et réapproprié par les colonisés, comme le montre le recyclage des cahiers coloniaux étudiés par Anne Bang et Didier Nativel. Aujourd'hui encore, le papier est peu produit en Afrique, et le papier indien inonde de nombreux marchés, comme le Nigeria (Mutai \& Brigaglia 2017). Le papier reste une denrée chère, notamment dans le monde de l'édition, qui le choisit en fonction du prix et des allègements fiscaux concédés par les pouvoirs publics, comme au Maroc (Cohen 2016 : 266). Les imprimeries se diffusèrent en Afrique à partir du XVIII ${ }^{\mathrm{e}}$ siècle et révolutionnèrent de nombreux champs de la vie intellectuelle et religieuse en Afrique (Chih, Seesemann \& Mayeur-Jaouen 2015). En 1767, une presse fut montée dans l'Île Maurice; en 1784, une autre fut mise en activité à Johannesbourg (Toussaint 1948 : 3). Les imprimeries sont également prises en main par les pouvoirs locaux, comme à Madagascar, en 1883, où le gouvernement royal publie son propre journal officiel (contribution de Didier Nativel). L'histoire de l'imprimerie préfigure alors celle de la presse coloniale et post-coloniale (Brennan 2013 ; Peterson, Hunter \& Newell 2016 ; Loimeier 2009), mais aussi de l'administration, de la bureaucratisation et des documents produits par ces dernières, comme les documents d'identité ou les visas (Awenengo Dalberto \& Banégas 2018).

5. Dans l'Indonésie néerlandaise, A. L. STOLER $(2019: 224)$ relève la citation ironique d'un journaliste métis critique vis-à-vis du pouvoir colonial : «On ne faisait que remplir des montagnes de paperasse pour le plus grand profit des fabriques de papier des Pays-Bas. » 
«Papiers, s'il vous plait». Le document et ses usages

Le lien entre papier et administration soulève la question des usages de l'objet écrit et des contextes où il circule. Si les contributions abordent surtout des objets faits de papier, leur nature diffère énormément, révélant la variété des situations dans lesquelles les objets écrits sont utilisés, à la fois par les populations lettrées, semi-lettrées et illettrées. Ainsi, Anouk Cohen et Alexandros Tsakos travaillent respectivement sur les textes liturgiques musulmans (le "Coran marocain ») et chrétiens (nubiens); Anne Bang et Didier Nativel décrivent les usages et le rôle des cahiers et des manuscrits intimes, religieux ou non, qui peuvent circuler de proche en proche, au sein d'une même confrérie religieuse ou être recyclés. Les lettres, laissez-passer et documents d'identité sont également des concentrés de techniques d'écriture, qui confèrent à l'objet et à leurs propriétaires un pouvoir, une protection dans leurs voyages et leurs déplacements (Didier Nativel et Rémi Dewière). La confiance dans les signes et marques de validation de l'artefact écrit, ainsi que les débats autour de leur légalité, sont centraux dans les relations commerciales à longue distance, comme dans le cas des suftajas discutées par Ghislaine Lydon.

L'articulation entre religion, économie, pouvoir et textualité est une thématique récurrente dans les contributions de ce dossier. Elle s'inscrit dans un courant de recherche extrêmement dynamique ces dernières années (Lydon 2009; Wion \& Bertrand 2011 ; Fitzgerald 2015 ; Kaiser \& Petitjean 2016 ; Potin 2016; Warscheid 2017). En particulier, la relation entre écriture et pouvoir est au cœur des «mondes de papier» (Mbodj-Pouye 2013 : 31 ; Rule \& Trotter 2014). Dans la lignée des recherches sur les écritures ordinaires (Bertrand 2015), pragmatiques (Wion, Barret \& Mbodj-Pouye 2016), ou bureaucratiques (Awenengo Dalberto \& Banégas 2018), plusieurs articles étudient les procédés techniques et les gestes liés à l'identification, à l'authenticité et à l'autorité des documents écrits. En particulier, l'article de Rémi Dewière montre que les variations des normes d'écriture épistolaires du pouvoir bornouan au XIX ${ }^{\mathrm{e}}$ siècle (nord Nigeria) permettent de comprendre la manière dont une administration sahélienne précoloniale travaille, mais aussi d'identifier ses acteurs et de les associer à une famille d'administrateurs proche du pouvoir depuis au moins l'époque moderne.

Le pouvoir ne se lit pas seulement dans les textes officiels ou ses réalisations les plus abouties. Les travaux d'Ann Laura Stoler (2019: 73-74) sur les archives coloniales ont montré que les marges et les notes de bas de pages révèlent les rapports de force, les économies émotionnelles ou les savoirs locaux qui irriguent les administrations, coloniales ou non. En Éthiopie moderne, les actes fonciers sont inscrits en marge des textes liturgiques (Bertrand \& Wion 2011) : 
ici, la marge est le refuge des textes officiels du pouvoir politique. Les actes sont alors pleinement intégrés dans la vie quotidienne des sociétés qui les produisent, prévenant ainsi les modifications et garantissant leur accès aux membres de l'institution (Wion 2017). Ces «transformations parasitaires » (Bertrand 2015 : 80) concernent à la fois les documents de pouvoir, mais également les textes intimes ou à visée éducative et religieuse : les travaux de Tal Tamari (2017) et Dmitry Bondarev (2017) montrent que le corps de texte n'est pas forcément l'élément le plus important des manuscrits arabes du Sahel, mais bien leurs marges, pour lesquelles le copiste laisse volontairement d'amples espaces vides pour des commentaires en arabe et en ajami, les langues africaines en caractères arabes. Parfois, une autre copie du même texte est réalisée pour ajouter de nouvelles notes marginales, avant que le manuscrit ne soit donné à un voisin ou un membre de sa famille, créant ainsi un réseau de copies en arabe et en ajami (Raia 2014, 2018).

L'article d'Anne Bang pose une question très proche : comment les manuscrits sont utilisés dans des cercles privés? Ses recherches historiques sur les manuscrits résonnent fortement avec les travaux de l'anthropologue Anouk Cohen, dont l'article traite des livres de Coran édités selon des règles définies par le pouvoir marocain. Elle montre comment les choix éditoriaux de ce texte au contenu fixe, familiers de la culture marocaine, visent à créer un rapport intime entre le livre saint et son propriétaire. Le Coran est à la fois utilisé comme support de l'écrit, mais également comme objet d'apparat ou, à l'opposé, intime et porteur d'une identité. Ces usages littéraires, paralittéraires et non littéraires de l'objet écrit remettent en question la séparation entre sociétés littéraires et sociétés non littéraires. Dans le sillage des travaux traitant des usages de l'écriture sur papier par les sociétés « aux lisières de l'alphabet » (Tóth 2001 ; Mbodj-Pouye 2013 ; Ware 2014) ou par les populations lettrées non scolarisées (Yusuf 2016), nous avons souhaité comprendre comment les supports de l'écrit circulent dans des sociétés largement « orales », et décrire les usages de l'écrit par des populations illettrées ou semi-lettrées.

Que ce soit durant la période précoloniale, coloniale ou post-coloniale, les situations de diglossie sont nombreuses dans le continent africain (Cohen 2016 : 30-31 ; Mbodj-Pouye 2013 : 31). Le fait de savoir lire et écrire est associé au pouvoir, comme dans le cas éthiopien (Crummey 2006), et l'écriture du pouvoir est une « langue étrangère » — dans le sens où elle n'est pas déchiffrable et où les langues utilisées sont allogènes, comme l'arabe, le français ou le grec. Dans ces contextes, le support matériel y gagne plus d'importance que le texte en lui-même. En effet, les artefacts écrits sont quotidiennement utilisés par des personnes qui ne savent ni lire, ni écrire. C'est le cas des écritures ordinaires, comme les actes juridiques ou les registres fonciers, mais aussi 
des lettres diplomatiques ou les documents d'identité, dont les marques de validation servent à circuler ou à conférer une autorité à une revendication exprimée à l'oral (article de D. Nativel, voir aussi Crummey 2006: 13) que ce soit avant, pendant ou après la colonisation. La place de l'objet écrit dans les sociétés africaines révèle également des réseaux, matérialisés par les mobilités de cour et d'archives, les alliances commerciales et diplomatiques ou, enfin, les communautés graphiques (Bertrand 2015 : 364). L'article de Rémi Dewière questionne les réseaux diplomatiques et les conventions normées au sein des administrations des États islamiques sahéliens, ainsi que les normes partagées durant les échanges épistolaires. Ghislaine Lydon parle, quant à elle, des textes qui circulent au sein des communautés marchandes afin de faciliter les transactions commerciales. À une tout autre échelle, Anne Bang questionne les « réseaux de pratiques » qui se constituent autour d'un texte en particulier, au sein de communautés religieuses ou d'amis.

\section{L'écrit entre sensorialité, esthétique et authenticité}

Remettre l'objet écrit au centre de l'enquête permet donc de dépasser la dichotomie entre écrit et oral. Si l'étude de Crummey (2006) sur les actes fonciers éthiopiens a montré que l'écrit n'est que la partie visible d'un ensemble de pratiques orales, l'artefact écrit est le reliquat de pratiques qui mêlent des paroles, des gestes, des éléments matériels et des corps, mais aussi des normes, des interdits et des transgressions. C'est ainsi que, dans le cas de la poésie swahilie en ajami, l'écriture est entrelacée avec le langage poétique, et vice versa (Vierke 2014) : étudier l'un permet de mieux comprendre l'autre, même si ce dernier a disparu. Dès lors, le parallèle avec l'archéologie prend tout son sens : à l'image des données archéologiques, les objets écrits qui nous sont aujourd'hui disponibles sont le résultat visible, passé à travers de nombreux filtres, d'un ensemble de pratiques littéraires et d'écriture, d'éléments matériels et immatériels (Bocquentin et al. 2010 : 160). Ainsi, la fragilité des supports de l'écrit laisse des traces et des indices précieux qui permettent d'explorer l'univers social et sensoriel dans lequel l'objet écrit s'insère. Or, les processus historiques, mais aussi différentes techniques de reproduction et de préservation (du travail du copiste à la numérisation des archives) sont autant de facteurs qui effacent des traces matérielles extrêmement volatiles et sortent les objets de leur contexte. L'écrit est alors reconditionné, réinscrit dans un nouveau format et dans un nouveau contexte, dégageant ainsi de nouvelles perceptions et stimulant de nouveaux sens. Ces processus valorisent, sélectionnent et préservent des éléments scripturaux et visuels, au dépend d'autres traces physiques qui disparaissent ou deviennent transparentes. 
La fragilité et la volatilité de ces traces amènent Didier Nativel à reprendre la phénoménologie de la perception de Maurice Merleau-Ponty pour définir la sensorialité comme un entre-deux entre perception et matérialité. Une perspective privilégiant la sensorialité du support écrit vise à donner « chair » aux documents, pour pouvoir ainsi reconstruire l'univers sensoriel et perceptif dans lequel ces textes sont incorporés. Il s'agit de voir comment l'objet écrit affecte les sujets dans leur rapport avec l'environnement. Didier Nativel conçoit la notion de « réversibilités documentaires », pour mettre en lumière comment le pouvoir des papiers d'identification de l'époque coloniale (du pass sud-africain aux cadernetas indigenas mozambicains ou kipande au Kenya) modifie le champ sensoriel de l'ambiance urbaine des villes coloniales. Le papier d'identification, les laissez-passer, sont des instruments du contrôle des corps, qui se chargent d'un pouvoir administratif et bureaucratique capable d'affecter le statut social et la vie intime et quotidienne des sujets.

L'objet écrit est aussi pris en compte dans son horizon performatif. L'analyse des cérémonies autour des textes font ressortir des bruits, des gestes et des odeurs qui accompagnent leur circulation ou leur archivage (Wion 2017). Ainsi, la vue, l'ouïe, l'odorat, le goût et le toucher provoquent des émotions qui ont été volontairement ou involontairement produites par les créateurs ou les commanditaires de l'objet. La dimension physique est reprise dans la contribution d'Alexandros Tsakos qui enquête sur la manière dont les sens sont mobilisés par l'objet écrit. En s'appuyant sur les sciences cognitives, sa contribution propose une histoire sociale des sens en Nubie chrétienne médiévale, en se basant sur la littérature religieuse, l'anthropologie et l'archéologie. De même, Anouk Cohen montre que les critères d'éditions des « Corans marocains » cherchent à privilégier une lecture et une intonation coranique proprement « marocaine ».

La dimension sensible du livre permet de toucher du doigt la question des représentations et du rôle symbolique de l'objet-livre, à partir de ses reproductions factices. Le livre feint, ou faux-livre, simule le livre sans l'être. Il peut remplir des fonctions esthétiques, ludiques, profanes, sacrées ou même scientifiques. Composé de matériaux divers, comme le bois ou la céramique, on le trouve dans toutes les sociétés lettrées et à toutes les époques, de l'Europe à l'époque moderne (Bernasconi 2017 : 164) aux salons marocains contemporains (Cohen 2016 : 96). Si nous nous sommes tenus à l'étude du papier, les questions soulevées par ces exemples ont alimenté notre réflexion : l'esthétique et la façon dont l'objet écrit produit un sens d'authenticité est centrale dans ce numéro. La qualité, la forme et la taille, tout comme les marques de validation, la calligraphie, le sceau ou l'espace entre les lignes, permettent d'identifier un document, mais contribuent également à provoquer des réactions à ces lecteurs 
à travers une visualité étroitement associée à un pouvoir (article de Rémi Dewière). Ces marques d'identification sont recyclées, recopiées, falsifiées ou réappropriées dans d'autres contextes et contribuent, dans de nombreuses sociétés africaines, à créer une esthétique particulière. Plus qu'une histoire de l'objet écrit, c'est une histoire de ces esthétiques et de ces communautés graphiques que nous souhaitons porter à travers ce numéro.

Spécialistes d'aires et d'époques variées, les auteurs de ce numéro travaillent sur les sociétés du continent africain, de l'Afrique du Nord à Madagascar en passant par la bande sahélienne. En questionnant la matérialité des sources textuelles, nous envisageons dépasser les bornes chronologiques, puisque les articles traitent aussi bien des périodes médiévales, modernes et contemporaines. Cette volonté de dépasser les barrières temporelles, géographiques et disciplinaires se reflète également dans le choix des recensions de ce numéro : du Pakistan contemporain à la France médiévale, du Yemen au Sahel, les ouvrages recensés touchent, de près ou de loin, aux thématiques qui nous ont été chères dans ce projet : si l'ensemble des contributions concernent l'Afrique, elles répondent à des questions plus larges sur la matérialité de l'écrit et de ses messages.

Institut des mondes africains (IMAF), Paris, France;

Institute of Advanced Studies, University of Warwick, Royaume-Uni.

BIBLIOGRAPHIE

Anheim É. \& Chastang P., 2009, «Les pratiques de l'écrit dans les sociétés médiévales $\left(\mathrm{VI}^{\mathrm{e}}-\mathrm{XIII}{ }^{\mathrm{e}}\right.$ siècle) $)$, Médiévales, $56: 5-10$.

Awenengo Dalberto S. \& Banégas R., 2018, «Citoyens de papier : des écritures bureaucratiques de soi en Afrique », Genèses, 112 (3) : 3-11.

Bausi A. ET AL. (EDS.), 2015, Comparative Oriental Manuscript Studies. An Introduction, Hambourg, Tredition.

- 2018, Manuscripts and Archives: Comparative Views on Record-keeping, Berlin, Walter de Gruyter. 
BERnASCONI G., 2017, «Transposition des formes matérielles et analogie : le cas des collections de bois en forme de livre au XVIII siècle », in S. ARCHAMbaUlT DE BeAune, L. Hilaire-PÉrez \& K. Vermeir (dir.), L'analogie dans les techniques, Paris, CNRS Éditions : 163-179.

Bertrand P., 2015, Les écritures ordinaires, Paris, Éditions de la Sorbonne.

Bertrand P. \& Wion A., 2011, « Production, Preservation, and Use of Ethiopian Archives (Fourteenth-Eighteenth Centuries) », Northeast African Studies, 11 (2): vii-xvi.

Bloom J., 2008, «Paper in Sudanic Africa », in S. Jeppie \& S. B. Diagne (eds.), The Meanings of Timbuktu, Cape Town, HSRC Press : 45-58.

BoCQUENTIN F. ET AL., 2010, «De la récurrence à la norme : interpréter les pratiques funéraires en préhistoire », Bulletins et mémoires de la Société d'anthropologie de Paris, 22 : 157-171.

BonacCORSI J., 2013, « Approches sémiologiques du web », in C. BARATs (dir.), Manuel d'analyse du web en Sciences humaines et sociales, Paris, Armand Colin : 125-146.

BONDAREV D., 2017, « Islamic Education and Ample Space Layout in West African Islamic Manuscripts », in A. Brigaglia \& M. NoBILI (eds.), The Arts and Crafts of Literacy: Islamic Manuscript Cultures in Sub-Saharan Africa, Berlin, De Gruyer : 105-142.

Bоотн M., 2001, «Woman in Islam : Men and the "Women's Press” in Turn-of-the-20thCentury Egypt», International Journal of Middle East Studies, 33 (2) : 171-201.

Brennan J. R., 2013, «Communications and Media in African History », in J. PARKeR \& R. REID (eds.), The Oxford Handbook of Modern African History, Oxford, Oxford University Press : 492-509.

Brigaglia A. \& Nobili M., 2017, The Arts and Crafts of Literacy. Islamic Manuscript Cultures in Sub-Saharan Africa, Berlin, De Gruyter.

CAVAllo G., 1983, Libri, scritture, scribi a Ercolano, Napoli, Gaetano Macchiaroli editore.

Chartier R. (DIR.), 1985, Pratiques de la lecture, Marseille, Rivages.

- 1987a, Les usages de l'imprimé (XVe-XIXe siècle), Paris, Fayard.

- 1987b, Lectures et lecteurs dans la France d'Ancien Régime, Paris, Éditions du Seuil.

- 1991, La correspondance : les usages de la lettre au $19^{e}$ siècle, Paris, Fayard.

Chin R, Seesemann R. \& Mayeur-Jaouen C. (eds.), 2015, Sufism, Literary Production, and Printing in the Nineteenth Century, Würzburg, Ergon Verlag.

Cohen A., 2016, Fabriquer le livre au Maroc, Paris, Karthala.

CRUMmeY D., 2006, «Literacy in an Oral Society : The Case of Ethiopian Land Records », Journal of African Cultural Studies, 18 (1) : 9-22. 
Davis C., Dick A. \& Le Roux E., 2018, «Introduction : Print Culture in Southern Africa », Journal of Southern African Studies, 44 (3) : 377-381.

Delmas A., 2017, « Writing in Africa. The Kilwa Chronicle and other Sixteenth Century Portuguese Testimonies », in A. Brigaglia \& M. NoBILI (eds.), The Arts and Crafts of Literacy. Islamic Manuscript Cultures in Sub-Saharan Africa, Berlin, De Gruyter : 181-206.

Delmas A. \& Penn N. (eds.), 2012, Written Culture in a Colonial Context. Africa and the Americas, 1500-1900, Leiden-Boston, Brill.

Denis J. \& Pontille D., 2012, «Travailleurs de l'écrit, matières de l'information », Revue d'anthropologie des connaissances, 6 (1) : 1-20.

DERAT M., 2018, L'énigme d'une dynastie sainte et usurpatrice dans le royaume chrétien d'Éthiopie du XIe au XIII ${ }^{e}$ siècle, Turnhout, Brepols.

Déroche F., 2000, Manuel de codicologie des manuscrits en écriture arabe, Paris, BnF Éditions.

- 2005, Islamic Codicology: an Introduction to the Study of Manuscripts in Arabic Script, London, al-Furqān Islamic Heritage Foundation.

Dewière R., 2017, Du lac Tchad à La Mecque. Le sultanat du Borno et son monde (XVI - $_{-}$ XVII ${ }^{e}$ siècle), Paris, Éditions de la Sorbonne.

FABRE D. (DIR.), 1993, Écritures ordinaires, Paris, P.O.L.

Fauvelle F.-X., Hirsch B. \& CheKroun A., 2017, « Le sultanat de l'Awfăt, sa capitale et la nécropole des Walasma“», Annales Islamologiques, 51 : 239-295.

Ficquet É. \& Mbodj-Pouye A., 2009, «Cultures de l'écrit en Afrique. Anciens débats, nouveaux objets », Annales. Histoire, Sciences Sociales, 64 (4) : 751-764.

FitzGerald T., 2015, « Reaching the Flocks : Literacy and the Mass Reception of Ottoman Law in the Sixteenth-Century Arab World », Journal of the Ottoman and Turkish Studies Association, 2 (1) : 5-20.

FreIsT D., à paraître, " "A Very Warm Surinam Kiss”. Staying Connected, Getting Engaged-Interlacing Social Sites of the Moravian Diaspora ", in D. FrEIST \& S. LACHEnicht (eds.), Connectiong Worlds and People. Early Modern Diasporas, London-New York, Routledge : 62-80.

Furet F. \& Ozouf J., 1977, Lire et écrire. L'alphabétisation des Français de Calvin à Jules Ferry, Paris, Éditions de Minuit.

Glasman J., 2012, «"Connaître papier”. Métiers de police et État colonial tardif au Togo », Genèses, 86 (1) : 37-54.

Goody J., 2007, Pouvoirs et savoirs de l'écrit, Paris, La Dispute. 
Hall N., 2000, « The Materiality of Letter Writing : A Nineteenth Century Perspective », in D. Barton \& N. Hall (eds.), Letter Writing as a Social Practice, Amsterdam, John Benjamins («Studies in Written Language and Literacy, $9 »): 83-108$.

HÉBrARD J., 1999, «Tenir un journal. L'écriture personnelle et ses supports », Cahiers RITM, $20: 9-50$.

Humbert G., 2001, « Le manuscrit arabe et ses papiers », REMMM, 99-100 : 55-77.

Hunwick J., 2005, Arabic Literature of Africa : Project and Publication, Northwestern, Northwestern University Program of African Studies (« Working Paper Series »).

Hutchinson S. E., 1996, Nuer Dilemmas : Coping with Money, War, and the State, Berkeley-Los Angeles-London, University of California Press.

JACOB C. (DIR.), 2011, Lieux de savoir 2. Les mains de l'intellect, Paris, Albin Michel.

JoHns A., 1998, The Nature of the Book. Print and Knowledge in the Making, Chicago, The University of Chicago Press.

Jungen C. \& RAYMOND C., 2012, «Introduction », Ateliers d'anthropologie, 36, $<$ http://journals.openedition.org/ateliers/9080>.

Kaiser W. \& Petitjean J. (eds.), 2016, «Litigation and the Elements of Proof in the Mediterranean $\left(16^{\text {th }}-19^{\text {th }}\right.$ C. $) »$, Quaderni Storici, 51 (3) : 619-776.

KRÄTli G. \& Lydon G. (EDS.), 2011, The Trans-Saharan Book Trade, Manuscript Culture, Arabic Literacy and Intellectual History in Muslim Africa, Brill, Leiden.

Lamberg J.-A., Ojala J., Peltoniemi M. \& SÄrkkä T. (eds.), 2012, The Evolution of Global Paper Industry, 1800-2050. A Comparative Analysis, Dordrecht-HeidelbergNew York-London, Springer.

Last M., 2008, « The Book in the Sokoto Caliphate », in S. Jeppie \& S. B. Diagne (eds.), The Meanings of Timbuktu, Cape Town, HSRC Press : 135-164.

Lefebvre C., 2009, «Itinéraires de sable. Parole, geste et écrit au Soudan central au XIX ${ }^{\mathrm{e}}$ siècle », Annales. Histoire, Sciences Sociales, 64 (4) : 797-824.

LE Roux, E., 2012, «Book History in the African World : The State of the Discipline », Book History, 15 : 248-300.

Levtzion N., 1965, «Early Nineteenth Century Arabic Manuscripts from Kumasi », Transactions of the Historical Society of Ghana, 8 : 99-119.

LoIMEIER R., 2009, Between Social Skills and Marketable Skills : the Politics of Islamic Education in $20^{\text {th }}$ Century Zanzibar, Brill, Boston.

LydON G., 2009, On Trans-Saharan Trails, Islamic Law, Trade Networks, and Cross-cultural Exchange in Nineteenth-Century Western Africa, Cambridge, Cambridge University Press. 
Mbodj-Pouye A., 2009, « Tenir un cahier dans la région cotonnière du Mali. Support d'écriture et rapport à soi », Annales, Histoire, Sciences Sociales, 64 (4) : 855-885.

— 2013, Le fil de l'écrit. Une anthropologie de l'alphabétisation au Mali, Lyon, ENS Éditions.

Mbodj-Pouye A. \& Fraenkel B., 2010, «Introduction. Les New Literacy Studies, jalons historiques et perspectives actuelles », Langage et société, 133 (3) : 7-24.

Mirza S., 2017, «The Visual Resonances of a Harari Qur'ān : An $18^{\text {th }}$ Century Ethiopian Manuscript and its Indian Ocean Connections », Afriques, 08, $<$ http://journals.openedition.org/afriques/2052>.

De Moraes Farias P. F., 2003, Arabic Inscription from the Republic of Mali, Epigraphy, Chronicles and Songhay-Tuareg History, Oxford, Oxford University Press.

Morelle L., 2009, «Usages et gestion de l'écrit documentaire (Occident, VIe-XII ${ }^{\mathrm{e}}$ siècle) : quelques considérations sur l'acte écrit ", L'autorité de l'écrit au Moyen Âge (OrientOccident) : XXXIX Congrès de la SHMESP (Le Caire, 30 avril-5 mai 2008), Paris, Éditions de la Sorbonne : 117-126.

Mutai M. B. \& Brigaglia A., 2017, «Kảana Umar's “CCI Quran”: The Making of a Bornuan Manuscript in the Twenty-First Century », in A. Brigaglia \& M. NoBILI (eds.), The Arts and Crafts of Literacy: Islamic Manuscript Cultures in Sub-Saharan Africa, Berlin, De Gruyer : 331-352.

NobILI M., 2011, « Arabic Scripts in West African Manuscripts : A Tentative Classification from the De Gironcourt Collection », Islamic Africa, 2 (1) : 105-133.

OchaŁA G., 2014, «Multilingualism in Christian Nubia : Qualitative and Quantitative Approaches », Dotawo : A Journal of Nubian Studies, 1 : 1-50.

O'FAhey R. S. \& ABu SAlim M., 1983, Land in Dar Fur : Charters and Documents from the Dar Fur Sultanate, Cambridge, Cambridge University Press.

PARKIN D., 2005, « Textile as Commodity, Dress as Text: Swahili Kanga and Women's Statements », in R. BARneS (ed.), Textiles in Indian Ocean Societies, London-New York, Routledge Curzon : 47-67.

Peterson D. R., 2004, Creative Writing: Translation, Bookkeeping, and the Work of Imagination in Colonial Kenya, Portsmouth, N. H., Heinemann Publishers.

Peterson D. R., Hunter E. \& Newell S. (EDS.), 2016, African Print Cultures : Newspapers and their Publics in the Twentieth Century, Ann Arbor, University of Michigan Press.

Petrucci A., 1979, Libri, scrittura e pubblico nel Rinascimento, Roma-Bari, Laterza.

- 2008, Scrivere lettere. Una storia plurimillenaria, Roma-Bari, Laterza.

Potin Y., 2016, «Le prix de l’écrit », Genèses, 105 (4) : 3-7. 
RAAPKe A., 2017, « The Pain of Senses Escaping. $18^{\text {th }}$ Century Europeans and the Sensory Challenges of the Caribbean », in D. HACKE \& P. Musselwhite (dir.), Empire of Senses. Sensory Practices and Modes of Perception in the Atlantic World, Leiden, Brill : 115-139.

RaIA A., 2014, «Remarks on Swahili Manuscripts in Arabic Script : Layout and Orthography of the Utendi wa Yusuf », in I. KRASKA-SzLENK \& B. WóJTOWICZ (eds.), Current Research in African Studies. Papers in Honour of Mwalimu Dr. Eugeniusz Rzewuski, Varsovie, Elipsa : 313-330.

- 2018, «A Mosaic of Scripts : Arabic Script in Africa from a Comparative Perspective », Quaderni di studi arabi, $12:$ 207-218.

Regourd A. (ED.), 2018, The Trade in Paper Marked with Non-Latin Characters/Le commerce des papiers à marques à caractères non latins, Leiden, Brill.

RicARD A., 1995, Littératures d'Afrique noire. Des langues aux livres, Paris, CNRS Éditions- Karthala.

Roche D., 1997, Histoire des choses banales. Naissance de la consommation dans les sociétés traditionnelles (XVII ${ }^{e}-X I X^{e}$ siècle), Paris, Fayard.

Rule J. \& Trotter B., 2014, A World of Paper. Louis XIV, Colbert de Torcy, and the Rise of the Information State, Montréal-Kingston, McGill-Queen's University Press.

Stoler A., 2019, Au cour de l'archive coloniale. Questions de méthode, Paris, Éditions de l'EHESS.

Stollberg-Rilinger B., 2008, « La communication symbolique à l'époque pré-moderne. Concepts, thèses, perspectives de recherche », Trivium, 2,

$<\mathrm{http}$ //journals.openedition.org/trivium/1152>.

Stolow J., 2010, Orthodox by Design. Judaism, Print Politics, and the ArtScroll Revolution, Berkeley, University of California Press.

Street B. (ED.), 1993, Cross-cultural Approaches to Literacy, Cambridge, Cambridge University Press.

TAMARI T., 2017, «Les manuscrits étudiés dans un cercle d'amis : analyse des gloses maninka et arabes du recueil BNF ms arabe 5674 », communication à la journée d'étude Paroles de Papier, organisée par S. Bruzzi \& Rémi Dewière, IMAf, Paris, 15 mars.

Thyrza Sparks R., 2013, «Re-writing the Script : Decoding the Textual Experience in the Bronze Age Levant (c.2000-1150 bc) », in K. Piquette \& R. Whitehouse (eds.), Writing as Material Practice. Substance, Surface and Medium, London, Ubiquity Press : 75-104.

Tо́тн I. G., 2001, «Une société aux lisières de l'alphabet. La paysannerie hongroise aux $\mathrm{XVII}^{\mathrm{e}}$ et XVIII ${ }^{\mathrm{e}}$ siècles », Annales. Histoire, Sciences Sociales, 56 (4) : 863-880. 
Toussaint A., 1948, «Les débuts de l'imprimerie aux Îles Mascareignes », Revue d'histoire des colonies, 35 (122) : 1-26.

VAn Dalen D., 2016, Doubt, Scholarship and Society in 17th-Century Central Sudanic Africa, Leiden, Brill.

VAn Damme S., 2015, «Un Ancien régime des sciences et des savoirs », in D. PeStre (dir.), Histoire des sciences et des savoirs, t. 1, Paris, Éditions du Seuil : 19-40.

Vaughan M., 2007, « Scarification in Africa : Re-reading Colonial Evidence », Cultural and Social History, 4 (3) : 385-400.

VIERKe C., 2014, «Akhi patia kalamu: Writing Swahili Poetry in Arabic Script», in M. Mumin \& K. Versteegh (eds.), The Arabic Script in Africa: Studies in the Use of a Writing System, Brill, Leiden : 319-339.

Walz T., 1985, "The Paper Trade of Egypt and the Sudan in the $18^{\text {th }}$ and $19^{\text {th }}$ Centuries ", in R. Hill, M. W. DAly \& T. WAlz (eds.), Modernization in the Sudan : Essays in Honor of Richard Hill, New York, L. Barber Press : 29-48.

WARE R., 2014, The Walking Qur'an: Islamic Education, Embodied Knowledge, and History in West Africa, Chapel Hill, North Carolina, The University of North Carolina Press.

WARSCHeID I., 2017, Droit musulman et société au Sahara prémoderne. La justice islamique dans les oasis du Grand Touat (Algérie) aux XVII $-X I X^{e}$ siècles, Leiden, Brill.

WiOn A., 2017, «"May the King Die !” Validation of Legal and Administrative Documents in Ethiopian Christian Culture $\left(15^{\text {th }}-19^{\text {th }} \mathrm{c}\right.$.) », communication à la journée d'étude Paroles de Papier, organisée par S. Bruzzi \& R. Dewière, IMAf, Paris, 15 mars.

Wion A., BArret S. \& Mbodj-Pouye A. (DIR.), 2016, «L'écrit Pragmatique africain », Afriques. Débats, méthodes et terrains, 7, <http://journals.openedition.org/ afriques/1941>.

Wion A. \& Bertrand P., 2011, « Production, Preservation and Use of Ethiopian Archives $\left(11^{\text {th }}-20^{\text {th }}\right.$ Centuries) : Introduction to the Special Issue », Northeast African Studies, 9 (2) : vii-xvi.

Yusuf H. A., 2016, Composing Egypt : Reading, Writing, and the Emergence of a Modern Nation, 1870-1930, Stanford, Stanford University Press. 